\title{
Values of PCT and STREM-1 combined with clinical pulmonary infection score for the prognostic evaluation of elderly patients with ventilator-associated pneumonia
}

\author{
Jianjun Wang ${ }^{1}$, Xiaoyan Zhu², Xiaoling Wang ${ }^{1}, \mathrm{Xi} \mathrm{Li}^{3^{*}}$ \\ 1. Cadre Ward Two, 900th Hospital of the Joint Logistics Team, China \\ 2. Tuberculosis, Meifeng Branch of 900th Hospital of the Joint Logistics Team, China \\ 3. Geriatrics, The Second Affiliated Hospital of Xi'an Jiaotong University, China
}

\begin{abstract}
Objective: To explore the values of PCT and STREM-1 combined with clinical pulmonary infection score (CPIS) for evaluating ventilator-associated pneumonia (VAP) in elderly patients. Methods: A total of 120 eligible patients were selected as VAP group, and 60 other patients without VAP were selected as control group. According to APACHE II scores, VAP group was divided into low-, medium-, and high-risk groups. The correlations of PCT, STREM-1, and CPIS with APACHE II score were analyzed. The values of PCT and STREM-1 combined with CPIS for evaluating prognosis were analyzed using ROC curves. Results: In the VAP group, serum PCT and STREM-1 levels and CPIS after mechanical ventilation significantly exceeded those of the control group and before mechanical ventilation $(P<0.05)$. These values were higher in the high-risk group than in medium-and low-risk groups $(P<0.05)$. They were positively correlated with APACHE II score $(P<0.001)$. These values of the death group were significantly higher than those of the survival group $1,3,5$, and $7 d$ after treatment $(P<0.05)$. AUC of PCT and STREM-1 combined with CPIS for assessing prognosis was $0.892(P<0.001)$. Conclusions: Serum PCT and STREM-1 levels and CPIS significantly increase in elderly patients with VAP, which rise with aggravation. Their combination has higher values for prognostic evaluation.
\end{abstract}

Keywords: procalcitonin, soluble triggering receptor expressed on myeloid cells-1, clinical pulmonary infection score, ventilator-associated pneumonia

Received: $15^{\text {th }}$ November 2021; Accepted: 13 ${ }^{\text {th }}$ January 2022; Published: $14^{\text {th }}$ January 2022

\section{Introduction}

Ventilator-associated pneumonia (VAP) refers to lung parenchymal infection occurring after mechanical ventilation for $48 \mathrm{~h}$, which is one of the common and severe complications during mechanical ventilation, with a mortality rate of up to $60 \%$ in elderly patients (1). Therefore, how to evaluate early the severity of VAP in elder- ly patients and to predict the clinical prognosis has important clinical significance for reducing mortality rate. Serum procalcitonin (PCT) has high sensitivity and specificity for the early diagnosis of infectious diseases, and it can also indicate the severity and prognosis of the disease (2). As a new inflammatory index, human soluble triggering receptor expressed on myeloid cells-1 (STREM-1) serves as a marker of bac-

\footnotetext{
* Corresponding author: Xi Li, Geriatrics, The Second Affiliated Hospital of Xi'an Jiaotong Universit, China. 
terial infection, and its level in serum is closely related to the severity of infection (3). The clinical pulmonary infection score (CPIS) is used to evaluate the infection in patients through clinical manifestations, imaging features, and microbiological indices, which is currently one of the commonly-used important indices evaluating the severity and prognosis of pulmonary infection (4).

Detection using a single index may be limited. Thereby motivated, serum PCT and STREM-1 expression levels were combined with CPIS in this study to explore the value for evaluating the prognosis of elderly patients with VAP.

\section{Methods}

\section{Clinical data}

A total of 120 critical elderly patients with VAP after mechanical ventilation in general ICU of our hospital from January 2018 to June 2020 were selected as VAP group, including 73 males and 47 females aged 62-86 years old, with an average of $(72.31 \pm 9.25)$ years. Other 60 elderly patients without VAP after mechanical ventilation for more than $48 \mathrm{~h}$ during the same period were selected as control group, including 36 males and 24 females aged $60-85$ years old, with an average of (71.68 \pm 8.97$)$ years. According to the acute physiology and chronic health evaluation II (APACHE II) score (5), the patients in the VAP group were divided into low-risk group (APACHE II score $<10$ points, $n=32$ ), medium-risk group (APACHE II score $=10-20$ points, $\mathrm{n}=43$ ), and high-risk group (APACHE II score $>20$ points, $n=45$ ). Based on the prognosis of patients after hospitalization for $30 \mathrm{~d}$, they were divided into survival group $(n=72)$ and death group $(n=48)$. This study was approved by the Ethics Committee of our hospital, and the test methods were strictly in accordance with the relevant regulations. All subjects and their families were informed of relevant rights and risks, and they voluntarily participated in the research and signed the informed consent.

After $1 \mathrm{~h}$ of non-invasive ventilation, intubation/ mechanical ventilation should be given when one of the following situations occurs: 1) Breathing or cardiac arrest; 2) $\mathrm{PaO}_{2} / \mathrm{FiO}_{2}<200$; 3) apnea or heart rate $<50 \mathrm{bpm}$, accompanied by disturbance of consciousness or wheezing; 4) mania that cannot be well controlled by sedatives; 5) severe dyspnea, accompanied by reverse chest and abdominal breathing or strong contraction of auxiliary respiratory muscles; 6) a large amount of aspiration or difficulty in managing respiratory secretions; 7) hypotension (systolic blood pressure $<90 \mathrm{mmHg}$ ) and no response to liquid or vasoactive drugs; 8) severe metabolic and (or) respiratory acidosis $(\mathrm{pH} \leq 7.20)$.

\section{Diagnostic criteria for $V A P$}

The diagnostic criteria for VAP are based on the Guidelines for the Diagnosis, Prevention and Treatment of Ventilator-Associated Pneumonia developed by the Chinese Medical Association in 2013 (6): After mechanical ventilation for $\geq 48$ $h$, new or progressive infiltration shadows in the lungs are shown in chest X-ray examination, and at least two of the following criteria are met: 1) Fever: The body temperature of patients rises $\left(>38^{\circ} \mathrm{C}\right)$; 2) Peripheral hemogram index white blood cells $>10.0 \times 10^{9} / \mathrm{L}$ or $\left.<4.0 \times 10^{9} / \mathrm{L} ; 3\right)$ Wet rales can be heard by auscultation of lungs, and a large number of purulent secretions can be seen in the respiratory tract; 4) New pathogenic bacteria appear in the respiratory secretions after culture.

\section{Inclusion and exclusion criteria}

Inclusion criteria: 1) VAP patients meeting the above diagnostic criteria; 2) those aged $\geq 60$ years old; 3 ) those receiving mechanical ventilation $\geq 48 \mathrm{~h}$.

Exclusion criteria: 1) Patients who failed to tolerate mechanical ventilation and quit the treatment; 
2) those who died within $3 \mathrm{~d}$ after mechanical ventilation; 3) those with infections in lungs or other sites before mechanical ventilation; 4) those complicated with malignant tumors or immune diseases; 5) those with a history of bone marrow transplantation, organ transplantation or heart bypass surgery; 6) those with incomplete data.

First, 128 VAP patients were enrolled according to the inclusion criteria. Then 1 case intolerable to mechanical ventilation, 2 cases who died within 3 days after mechanical ventilation, 1 case with pulmonary infection before mechanical ventilation, 1 case complicated with lung cancer, 1 case who had received heart bypass surgery, and 2 cases with incomplete medical records were excluded. Finally, 120 patients were included in this study.

\section{Collection of baseline clinical data}

The basic clinical data of all patients were collected, including age, gender, body mass index (BMI), APACHE II score, duration of mechanical ventilation before diagnosis with VAP, length of stay in ICU, and underlying diseases (respiratory failure, COPD, cerebrovascular disease, cardiovascular disease, sepsis, type 2 diabetes mellitus, hypertension, chronic renal insufficiency, and digestive tract disease).

\section{Sample collection and detection}

Before and after mechanical ventilation, $4 \mathrm{~mL}$ of fasting venous blood was drawn from each subject, placed in a test tube containing heparin sodium, and centrifuged at 3,000 rpm for $10 \mathrm{~min}$. The supernatant was collected and stored in a refrigerator at $4^{\circ} \mathrm{C}$, followed by detection within 1 h. The levels of serum PCT and STREM-1 were detected through enzyme-linked immunosorbent assay using kits (Rapidbio, USA) in strict accordance with the instructions in the Laboratory Department of our hospital. The normal reference ranges were as follows: $\mathrm{PCT}<0.05 \mathrm{ng} /$ $\mathrm{mL}$, and STREM-1 $<92.00 \mathrm{ng} / \mathrm{L}$. The patients were treated with anti-infective therapy using the same measures from $1 \mathrm{~d}$ after diagnosis with VAP. Venous blood was drawn from patients at $1,3,5$, and $7 \mathrm{~d}$ of treatment, and then the serum PCT and STREM-1 expression levels were detected, and CPIS was calculated.

\section{CPIS}

CPIS was evaluated based on the following indices (7): 1) Body temperature: $36-38^{\circ} \mathrm{C}=0$ points, $38-39^{\circ} \mathrm{C}=1$ point, $>39^{\circ} \mathrm{C}$ or $\angle 36^{\circ} \mathrm{C}=2$ points; 2) White blood cell count: $4-11 \times 10^{9} / \mathrm{L}=0$ points, $11-17 \times 10^{9} / \mathrm{L}=1$ point, $<4 \times 10^{9} / \mathrm{L}$ or $>17 \times 10^{9} / \mathrm{L}=2$ points; 3) Tracheal secretions (character and number of aspirates at $24 \mathrm{~h}$ ): no or a little sputum $=0$ points, moderate-large amount of non-purulent sputum $=1$ point, moderate-large amount of purulent sputum $=2$ points; 4) Oxygenation indices $\left(\mathrm{PaO}_{2} / \mathrm{FiO}_{2}, \mathrm{kPa}\right)$ : $>33=0$ points, $<33=2$ points; 5) Infiltration shadows in chest $\mathrm{X}$-ray: none $=0$ points, patchy shadows $=1$ point, confluent patchy shadows $=2$ points; 6) Tracheal aspirate culture or sputum culture: no growth of pathogenic bacteria $=0$ points, growth of pathogenic bacteria $=1$ point; the same bacteria were obtained in both tracheal aspirate and sputum, or the Gram staining result was consistent with the culture result $=2$ points. The highest score was 12 points, and the score $\geq 6$ points indicated a high risk of death, or the lower score indicated relieved conditions.

\section{Statistical analysis}

SPSS 19.0 software was used for statistical analysis. All data were tested for homogeneity of variance and normal distribution. Normally distributed measurement data were expressed as mean \pm standard deviation ( $~ \chi \pm s$ ), and numerical data were expressed as frequency or rate. The independent-samples $t$ test and $\chi^{2}$ test were performed for intergroup comparison. The correlations of PCT, STREM-1, and CPIS with the APACHE II score were explored through 
Spearman's analysis. Receiver operating characteristic (ROC) curves were plotted based on the prognosis of the 120 included VAP patients after 30 days of hospitalization to assess the value of PCT and STREM-1 combined with CPIS. Onetailed $\mathrm{P}<0.05$ suggested a statistically significant difference.

\section{Results}

\section{Baseline clinical data}

There were no statistically significant differences in age, gender, BMI, APACHE II score, duration of mechanical ventilation, length of stay in ICU and underlying diseases between the two groups $(\mathrm{P}>0.05)$ (Table 1).

\section{PCT, STREM-1 and CPIS before and after mechanical ventilation}

There were no significant differences in serum PCT and STREM-1 expression levels and CPIS between the two groups before mechanical ventilation, and the same was true in the control group before and after mechanical ventilation $(\mathrm{P}>0.05)$. In the VAP group, the serum PCT and STREM-1 expression levels and CPIS after mechanical ventilation were significantly higher than those before mechanical ventilation and those after mechanical ventilation in the control group ( $\mathrm{P}<0.05)$ (Table 2).

\section{PCT, STREM-1, and CPIS after mechanical ventilation in high-, medium-, and low-risk groups}

After mechanical ventilation, the serum PCT and STREM-1 expression levels and CPIS were significantly higher in the high-risk group than those in the medium- and low-risk groups, while they were also significantly higher in the medium-risk group than those in the low-risk group $(\mathrm{P}<0.05)$. The serum PCT and STREM-1 expression levels and CPIS all had significant positive correlations with the APACHE II score $(r=0.845,0.800$ and 0.782, $\mathrm{P}<0.001$ ) (Table 3 and Figure 1).

\section{PCT, STREM-1, and CPIS at different time points after treatment in survival and death groups}

The serum PCT and STREM-1 expression levels and CPIS in the death group were significantly

Table 1. Baseline clinical data [ $\chi \pm \mathbf{s}, \mathbf{n}(\%)]$

\begin{tabular}{|c|c|c|c|c|}
\hline Index & VAP group $(n=120)$ & Control group $(n=60)$ & $t / \chi^{2}$ & $\mathbf{P}$ \\
\hline Age (Y) & $72.31 \pm 9.25$ & $71.68 \pm 8.97$ & 0.435 & 0.664 \\
\hline Male $[\mathrm{n}(\%)]$ & $73(60.83)$ & $36(60.00)$ & 0.484 & 0.486 \\
\hline BMI $\left(\mathrm{kg} / \mathrm{m}^{2}\right)$ & $22.47 \pm 2.32$ & $22.69 \pm 2.16$ & 0.613 & 0.540 \\
\hline APACHE II score (point) & $18.50 \pm 5.20$ & $18.40 \pm 5.90$ & 0.116 & 0.908 \\
\hline Duration of mechanical ventilation $(\mathrm{d})$ & $13.20 \pm 3.82$ & $13.30 \pm 3.96$ & 0.137 & 0.891 \\
\hline Length of stay in ICU (d) & $31.34 \pm 8.53$ & $32.56 \pm 9.47$ & 0.872 & 0.385 \\
\hline \multicolumn{5}{|l|}{ Underlying disease } \\
\hline Respiratory failure $[\mathrm{n}(\%)]$ & $39(32.50)$ & $19(31.67)$ & 0.013 & 0.910 \\
\hline COPD [n(\%)] & $21(17.50)$ & $6(10.00)$ & 1.765 & 0.184 \\
\hline Cerebrovascular disease $[\mathrm{n}(\%)]$ & $20(16.67)$ & $12(20.00)$ & 0.304 & 0.581 \\
\hline Cardiovascular disease $[\mathrm{n}(\%)]$ & $9(7.50)$ & $6(10.00)$ & 0.327 & 0.567 \\
\hline Sepsis $[\mathrm{n}(\%)]$ & $6(5.00)$ & $4(6.67)$ & 0.212 & 0.645 \\
\hline Type 2 diabetes mellitus [n(\%)] & $7(5.83)$ & $4(6.67)$ & 0.048 & 0.826 \\
\hline Hypertension $[\mathrm{n}(\%)]$ & $7(5.83)$ & $5(8.33)$ & 0.402 & 0.526 \\
\hline Chronic renal insufficiency $[\mathrm{n}(\%)]$ & $7(5.83)$ & $3(5.00)$ & 0.053 & 0.818 \\
\hline Digestive tract disease $[\mathrm{n}(\%)]$ & $4(3.33)$ & $1(1.67)$ & 0.411 & 0.521 \\
\hline
\end{tabular}


Table 2. PCT, STREM-1, and CPIS before and after mechanical ventilation ( $\chi \pm s$ )

\begin{tabular}{lcccc}
\hline \multirow{2}{*}{ Index } & \multicolumn{2}{c}{ VAP group (n=120) } & \multicolumn{2}{c}{ Control group (n=60) } \\
\cline { 2 - 5 } & $\begin{array}{c}\text { Before mechanical } \\
\text { ventilation }\end{array}$ & $\begin{array}{c}\text { After mechanical } \\
\text { ventilation }\end{array}$ & $\begin{array}{c}\text { Before mechanical } \\
\text { ventilation }\end{array}$ & $\begin{array}{c}\text { After mechanical } \\
\text { ventilation }\end{array}$ \\
\hline PCT $(\mathrm{ng} / \mathrm{mL})$ & $0.54 \pm 0.08$ & $3.13 \pm 0.47^{* \#}$ & $0.53 \pm 0.09$ & $0.54 \pm 0.07$ \\
\hline STREM-1 $(\mathrm{ng} / \mathrm{L})$ & $98.04 \pm 22.47$ & $154.12 \pm 42.30^{* \#}$ & $97.62 \pm 21.25$ & $100.58 \pm 19.68$ \\
\hline CPIS & $5.18 \pm 2.66$ & $7.65 \pm 3.01^{* \#}$ & $5.19 \pm 2.27$ & $5.84 \pm 2.30$ \\
\hline
\end{tabular}

${ }^{*} \mathrm{P}<0.05$ vs. before mechanical ventilation, ${ }^{*} \mathrm{P}<0.05$ vs. control group.

Table 3. PCT, STREM-1, and CPIS after mechanical ventilation in high-, medium-, and low-risk groups $(\chi \pm \mathrm{s})$

\begin{tabular}{lccc}
\hline Group & PCT $(\mathbf{n g} / \mathbf{m L})$ & STREM-1 (ng/L) & CPIS \\
\hline Low-risk $(\mathrm{n}=32)$ & $1.84 \pm 0.49$ & $92.46 \pm 16.59$ & $4.22 \pm 1.21$ \\
\hline Medium-risk $(\mathrm{n}=43)$ & $2.89 \pm 0.53^{*}$ & $135.93 \pm 20.13^{*}$ & $6.19 \pm 1.59^{*}$ \\
\hline High-risk $(\mathrm{n}=45)$ & $3.62 \pm 0.60^{* \#}$ & $161.02 \pm 19.30^{* \#}$ & $8.28 \pm 1.58^{* \#}$ \\
\hline
\end{tabular}

${ }^{*} \mathrm{P}<0.05$ vs. low-risk group, ${ }^{*} \mathrm{P}<0.05$ vs. medium-risk group.

higher than those in the survival group at 1, 3, 5, and $7 \mathrm{~d}$ of treatment $(\mathrm{P}<0.05)$. The above indices significantly rose with the prolongation of treatment time in death group $(\mathrm{P}<0.05)$, while they reached the peak at $5 \mathrm{~d}$ of treatment and then significantly declined at $7 \mathrm{~d}$ in the survival group $(\mathrm{P}<0.05)$. The serum PCT, STREM-1 expression levels and CPIS of the control group continuously decreased at $1,3,5$, and $7 \mathrm{~d}$ of treatment (Table 4).

Values of PCT and STREM-1 combined with CPIS for evaluating prognosis of VAP patients ROC curves were plotted based on the prognosis of the 120 included VAP patients after 30 days of hospitalization. The optimal cut-off values of PCT, STREM-1, and CPIS for assessing the prognosis of VAP patients were $2.56 \mathrm{ng} /$
$\mathrm{mL}, 121.34 \mathrm{ng} / \mathrm{L}$, and 6.13 points, respectively, and the area under the curve (AUC) was 0.805 , 0.821 , and 0.793 , respectively. The AUC of the combination of the three was 0.892 (95\% CI: $0.816-0.982, \mathrm{P}<0.001$ ), displaying a higher value for prognostic evaluation (Table 5 and Figure 2).

\section{Discussion}

VAP is a common infectious disease in ICU usually caused by bacteria, which frequently occurs in patients receiving invasive mechanical ventilation. During mechanical ventilation, the normal defense barrier of the respiratory tract is destroyed due to tracheotomy or tracheal intubation, thus damaging the normal airway muco-
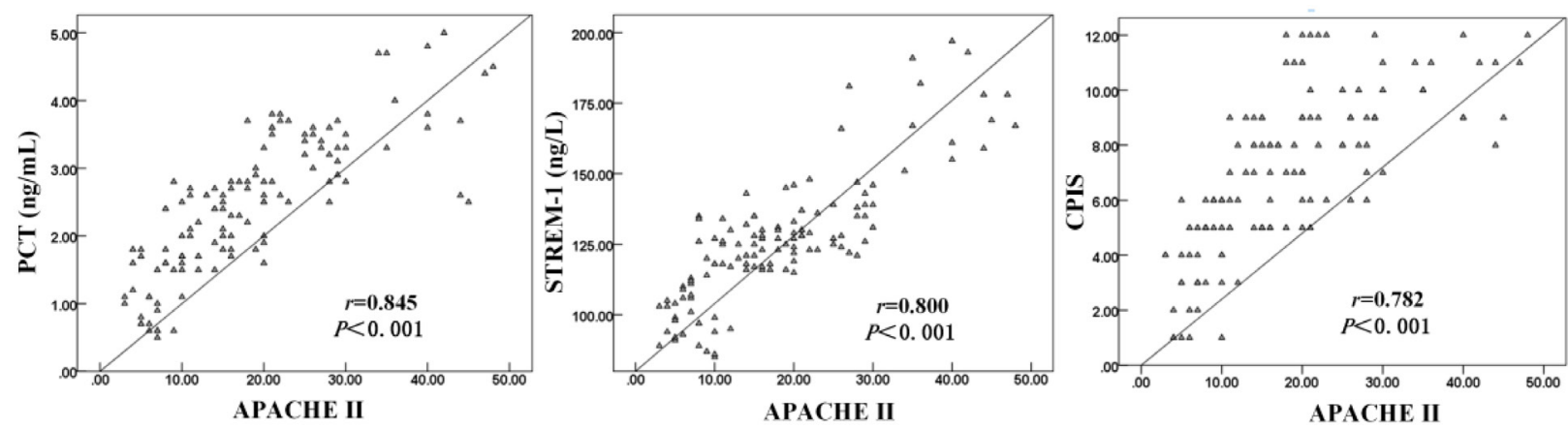

Fig. 1. Correlations of PCT, STREM-1, and CPIS with APACHE II score in VAP patients. 
Table 4. PCT, STREM-1, and CPIS at different time points after treatment in survival and death groups

\begin{tabular}{lccccc}
\multicolumn{7}{c}{$(\boldsymbol{C} \chi \mathbf{s})$} \\
\hline Index & Group & $\mathbf{1 ~ d}$ & $\mathbf{3 ~ d}$ & $\mathbf{5 ~ d}$ & $\mathbf{7 ~ d}$ \\
\hline PCT $(\mathrm{ng} / \mathrm{mL})$ & Survival $(\mathrm{n}=72)$ & $2.53 \pm 0.49$ & $2.84 \pm 0.47^{\#}$ & $3.18 \pm 0.42^{\#}$ & $2.62 \pm 0.53^{\#}$ \\
\hline & Death $(\mathrm{n}=48)$ & $3.89 \pm 0.62^{* \#}$ & $4.72 \pm 0.73^{* \#}$ & $5.57 \pm 0.81^{* \#}$ & $6.49 \pm 0.88^{* \#}$ \\
\hline & Control $(\mathrm{n}=60)$ & $1.54 \pm 0.12$ & $1.12 \pm 0.14$ & $1.03 \pm 0.11$ & $0.45 \pm 0.05$ \\
\hline STREM-1 $(\mathrm{ng} / \mathrm{L})$ & Survival $(\mathrm{n}=72)$ & $106.46 \pm 28.47$ & $132.12 \pm 16.30^{\#}$ & $156.02 \pm 17.25^{\#}$ & $128.58 \pm 16.53^{\#}$ \\
\hline & Death $(\mathrm{n}=48)$ & $163.93 \pm 25.86^{* \#}$ & $178.05 \pm 15.42^{* \#}$ & $189.26 \pm 17.31^{* \#}$ & $204.50 \pm 16.49^{* \#}$ \\
\hline CPIS & Control $(\mathrm{n}=60)$ & $71.54 \pm 12.13$ & $62.43 \pm 6.25$ & $51.24 \pm 3.87$ & $43.28 \pm 3.11$ \\
\hline & Survival $(\mathrm{n}=72)$ & $6.42 \pm 1.01$ & $7.15 \pm 1.23^{\#}$ & $9.39 \pm 1.27^{\#}$ & $7.64 \pm 1.21^{\#}$ \\
\hline & Death $(\mathrm{n}=48)$ & $8.59 \pm 1.48^{* \#}$ & $10.24 \pm 1.36^{* \#}$ & $12.01 \pm 1.73^{* \#}$ & $13.35 \pm 1.98^{* \#}$ \\
\hline
\end{tabular}

${ }^{*} \mathrm{P}<0.05$ vs. survival group, ${ }^{\#} \mathrm{P}<0.05$ vs. the previous time point within the group.

Table 5. Values of PCT and STREM-1 combined with CPIS for prognostic evaluation of VAP patients

\begin{tabular}{lcccccc}
\hline Diagnostic index & Cut-off & AUC & $\mathbf{9 5 \%}$ CI & P & Sensitivity (\%) & Specificity (\%) \\
\hline PCT $(\mathrm{ng} / \mathrm{mL})$ & 1.97 & 0.805 & $0.783-0.832$ & $<0.001$ & 85.41 & 82.57 \\
\hline STREM-1 (ng/L) & 121.34 & 0.821 & $0.714-0.846$ & $<0.001$ & 81.22 & 88.13 \\
\hline CPIS & 6.13 & 0.793 & $0.735-0.857$ & $<0.001$ & 84.01 & 74.01 \\
\hline Combination & - & 0.892 & $0.816-0.982$ & $<0.001$ & 94.28 & 91.45 \\
\hline
\end{tabular}

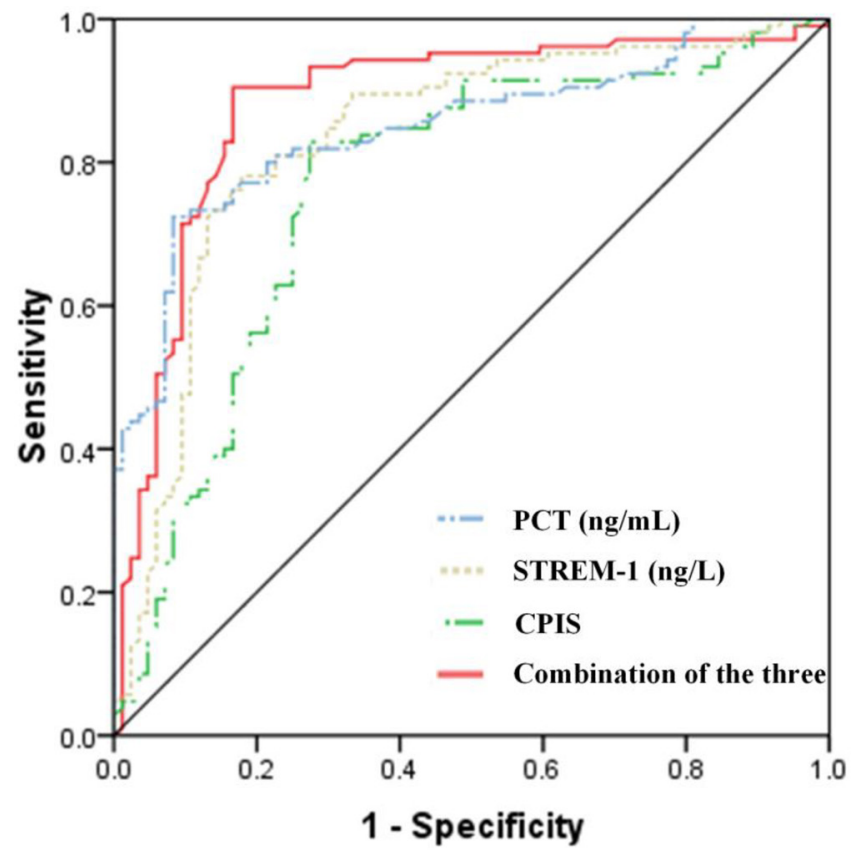

Fig. 2. ROC curve analysis results of values of PCT and STREM-1 combined with CPIS for prognostic evaluation of VAP patients. 
sa, and weakening the airway ciliary clearance ability. As a result, bacteria easily colonize and reproduce at the affected site, and then enter the lower respiratory tract from the upper respiratory tract, inducing VAP (8). At present, the clinical diagnosis of VAP is mainly based on clinical symptoms and signs, lung imaging, and sputum bacteriological test, which, however, have low specificity and cannot accurately determine bacterial, fungal, and viral infections. Moreover, the bacteriological test lasts for a long time, delaying the treatment (9).

It was found in previous clinical practice that PCT can be used to diagnose early potential infection in patients with VAP more accurately (10). PCT is produced by thyroid $\mathrm{C}$ cells and possesses no physiological activity, so its content in plasma is extremely low under normal physiological conditions. However, in the case of severe bacterial and parasitic infections and multiple organ failure, PCT can be synthesized and secreted by other tissues and organs, such as lung, liver, kidney, and adipose or muscle tissues, manifested as the ectopic production of PCT and abnormal elevation of its level, which is related to the severity and prognosis of infection (11). Wongsurakiat et al. showed that if the PCT level in patients was higher at 1-7 d after the occurrence of VAP, the risk of poor prognosis was higher (12). Coelho et al. assessed the dynamic changes in the serum PCT level at 1, 3, and $7 \mathrm{~d}$ after the occurrence of VAP, and confirmed that PCT could be used to predict the prognosis of patients with VAP (13). STREM-1 is a soluble inflammatory mediator associated with inflammation. During infection, STREM-1 can be released into the blood, body fluid and alveolar lavage fluid, which is closely related to the severity of infection. In particular, the level of STREM-1 in the serum of patients with sepsis and in the bronchoalveolar lavage fluid of patients with pneumonia significantly rises. Therefore, STREM-1 can be used for clinical diagnosis and prognostic evaluation
(14). Moralesortiz et al. found through detection of STREM-1 in the alveolar lavage fluid of patients clinically suspected of pulmonary infection with infiltration shadows in both lungs that the concentration of STREM-1 in patients with bacterial and fungal infections was higher than that in patients with atypical pathogen and viral infections, and its sensitivity and specificity in diagnosing bacterial or fungal infection were $86 \%$ and $90 \%$, respectively (15). Van Oort et al. detected STREM-1 in the exhaled air condensate from the ventilator of VAP patients, and showed that STREM-1 was significant to the diagnosis of VAP (16).

In the present study, the levels of serum PCT and STREM-1 after mechanical ventilation in the VAP group were far higher than those in the control group, and the more severe the condition of disease, the higher the expression levels of PCT and STREM-1. Both PCT and STREM-1 had significant positive correlations with the APACHE II score, indicating that PCT and STREM-1 can reflect the severity of VAP. Furthermore, the results revealed that the levels of PCT and STREM-1 in the death group were far higher than those in the survival group. With PCT $>1.97 \mathrm{ng} / \mathrm{mL}$ and STREM-1 >121.34 ng/ $\mathrm{mL}$ as the diagnostic criteria for poor prognosis, the area under the ROC curve was larger, and both sensitivity and specificity in the diagnosis of VAP were higher, demonstrating that PCT and STREM-1 can be used as diagnostic indices for the prognosis of VAP patients.

CPIS consists of body temperature, white blood cell count, respiratory secretions, oxygenation index, chest X-ray, and etiological data of airway secretions, which has been widely applied to determine the severity and evaluate the prognosis of lung diseases. It has been proven that the higher CPIS corresponds to the more severe pulmonary infection, worse prognosis and also a higher mortality rate (17). In this study, it was found that CPIS gradually rose with the wors- 
ening of condition in patients, which had a significant positive correlation with the APACHE II score, indicating that CPIS can well predict the potential occurrence of VAP and preliminarily reflect the infection status in patients. However, the specificity of CPIS alone in assessing the prognosis of patients is low. Its specificity was $74.01 \%$ in this study, similar to the finding of Elgazzar et al. that the diagnostic sensitivity and specificity of CPIS $>6.2$ points for VAP were $80.4 \%$ and $77.3 \%$, respectively (18). Therefore, the combination of CPIS with PCT, STREM-1 and other indices able to reflect the severity of the disease may be more conducive to evaluating the severity and prognosis of VAP in the elderly. In this study, the results revealed that the area under the ROC curve of serum PCT and STREM-1 expression levels combined with CPIS for assessing the prognosis of patients was far larger than that of a single index, and the sensitivity and specificity were $94.28 \%$ and $91.45 \%$, respectively. Therefore, the combination of the three indices has a higher value for evaluating the prognosis of VAP patients.

\section{Conclusion}

In conclusion, serum PCT and STREM-1 expression levels and CPIS are all significantly increased in VAP patients, and they rise with the increasing severity of the disease. The combination of the three has higher values for the prognostic evaluation of elderly patients with VAP. Regardless, this study has limitations. The findings herein are based on the experience of a single center, which should be verified by performing multi-center studies with larger sample sizes.

\section{Authors' contribution}

$\mathrm{Xi}$ Li designed this study and prepared this manuscript;

Xi Jianjun Wang, Xiaoyan Zhu and Xiaoling
Wang collected and analyzed clinical data. All authors have approved the submission and publication of this manuscript

\section{Conflict of interest}

None to declare.

\section{References}

1. Wood GC, Jonap BL, Maish III GO, Magnotti LJ, Swanson JM, Boucher BA, et al. Treatment of Achromobacter Ventilator-Associated Pneumonia in Critically Ill Trauma Patients. Ann Pharmacother. 2018;52:1205. DOI: $10.1177 / 1060028017730838$

2. Re MF, Rocchetti NS, Settecase CJ, Bagilet DH. Diagnostic value of procalcitonin in ventilator-associated pneumonia. Med Clin. 2019;152:216-21. DOI: 10.1016/j.medcle.2019.01.011

3. Li C, Zhu L, Gong X, Xu Z, Liu Y, Zhang M, et al. Soluble triggering receptor expressed on myeloid cells 1 as a useful biomarker for diagnosing ventilator associated pneumonia after congenital cardiac surgery in children. Exp Ther Med. 2019;17:147-52. DOI: 10.3892/ etm.2018.6905

4. Iosifidis E, Pitsava G, Roilides E. Ventilator-associated pneumonia in neonates and children: a systematic analysis of diagnostic methods and prevention. Future Microbiol. 2018;13:1431-46. DOI: 10.2217/fmb-20180108

5. Fu CM, Chang CH, Fan PC, Tsai MH, Lin SM, Kao KC, et al. Prognosis of critically ill cirrhotic versus non-cirrhotic patients: a comprehensive score-matched study. BMC Anesthesiol. 2014;14:123. DOI: 10.1186/14712253-14-123

6. Intensive Care Medicine Branch of Chinese Medical Association. [Guidelines for the Diagnosis, Prevention and Treatment of Ventilator-Associated Pneumonia (2013)]. Chin J Intern Med. 2013;52:524-43.

7. Gaudet A, Martin-Loeches I, Povoa P, Rodriguez A, Salluh J, Duhamel A, et al. Accuracy of the clinical pulmonary infection score to differentiate ventilator-associated tracheobronchitis from ventilator-associated pneumonia. Ann Intens Care. 2020;10:101. DOI: 10.1186/s13613-020-00721-4

8. Bos LD, Kalil AC. Changes in lung microbiome do not explain the development of ventilator-associated 
pneumonia. Intens Care Med. 2019;45:1133-5. DOI: 10.1007/s00134-019-05691-1

9. De Neef M, Bakker L, Dijkstra S, Raymakers-Janssen P, Vileito A, Ista E. Effectiveness of a Ventilator Care Bundle to Prevent Ventilator-Associated Pneumonia at the PICU: A Systematic Review and Meta-Analysis. Pediatr Crit Care Med. 2019;20:474-80. DOI: 10.1097/ PCC.0000000000001862

10. Wang Q, Hou D, Wang J, An K, Han C, Wang C. Procalcitonin-guided antibiotic discontinuation in ventilator-associated pneumonia: a prospective observational study. Infect Drug Resist. 2019;12:815-24. DOI: 10.2147/IDR.S190859

11. Beye F, Vigneron C, Dargent A, Prin S, Andreu P, Large A, et al. Adhering to the procalcitonin algorithm allows antibiotic therapy to be shortened in patients with ventilator-associated pneumonia. J Crit Care. 2019;53:12531. DOI: 10.1016/j.jcrc.2019.05.022

12. Wongsurakiat $\mathrm{P}$, Tulatamakit $\mathrm{S}$. Clinical pulmonary infection score and a spot serum procalcitonin level to guide discontinuation of antibiotics in ventilator-associated pneumonia: a study in a single institution with high prevalence of nonfermentative gram-negative bacilli infection. Ther Adv Respir Dis. 2018;12:1-13. DOI: $10.1177 / 1753466618760134$

13. Coelho L, Rabello L, Salluh J, Martin-Loeches I, Rodriguez A, Nseir S, et al. C-reactive protein and procalcitonin profile in ventilator-associated lower respiratory infections. J Crit Care. 2018;48:385-9. DOI: 10.1016/j. jcrc.2018.09.036

14. Edel Y, Kliminski V, Pokroy-Shapira E, Oren S, Lazar AD, Basson YP, et al. Elevated plasma level of soluble triggering receptor expressed on myeloid cells- 1 is associated with inflammation activity and is a potential biomarker of thrombosis in primary antiphospholipid syndrome. Arthritis Res Ther. 2019;21:1-10. DOI: 10.1186/s13075-018-1779-5

15. Moralesortiz J, Rondina MT, Brown SM, Grissom C, Washington AV. High Levels of Soluble Triggering Receptor Expressed on Myeloid Cells-Like Transcript (TLT)-1 Are Associated with Acute Respiratory Distress Syndrome. Clin Appl Thromb Hemost. 2018;24:1122-7. DOI: 10.1177/1076029618774149

16. Van Oort PM, Bos LD, Povoa P, Ramirez P, Torres A, Artigas A, et al. Soluble urokinase plasminogen activator receptor for the prediction of ventilator-associated pneumonia. ERJ Open Res. 2019;5(1):00212-2018. DOI: $10.1183 / 23120541.00212-2018$

17. Chen C, Yan M, Hu C, Lv X, Zhang H, Chen S. Diagnostic efficacy of serum procalcitonin, C-reactive protein concentration and clinical pulmonary infection score in Ventilator-Associated Pneumonia. Med Sci. 2018;34:26-32. DOI: 10.1051/medsci/201834f105

18. Elgazzar AE, Hosny H, Elkhateeb TH. Procalcitonin and clinical pulmonary infection score as predictors of stroke-associated pneumonia: a prospective observational single-center study. Egypt J Chest Dis Tuberc. 2017;2090:9950. 